\title{
¿CÓMO ALIMENTAR A LOS NIÑOS? LA PRÁCTICA DE CONDUCTAS ALIMENTARIAS SALUDABLES DESDE LA INFANCIA
}

\section{HOW TO FEED CHILDREN? HEALTHY EATING BEHAVIORS STARTING AT CHILDHOOD}

\author{
Maureen M. Black ${ }^{1, a}$, Hilary M. Creed-Kanashiro ${ }^{2, b}$
}

Department of Pediatrics, University of Maryland School of Medicine. Baltimore, EE. UU.

Instituto de Investigación Nutricional. Lima, Perú.

Psicóloga doctora en Filosofía, ${ }^{b}$ Nutricionista maestra en Filosofía

Recibido: 24-07-12; Aprobado: 29-08-12

\begin{abstract}
RESUMEN
Las intervenciones para prevenir la desnutrición o el exceso de peso en los niños se enfocan en la dieta, y dan poca atención a los comportamientos de las personas responsables de su cuidado. En sus primeros dos años los niños adoptan prácticas establecidas en su entorno y las conductas de sus cuidadores, los cuales constituyen patrones alimentarios que persistirán a lo largo de su vida. Así, los niños y sus cuidadores establecen una relación en que reconocen, interpretan y responden a las señales de comunicación verbal y no verbal. Alimentar al niño adoptando una conducta "responsiva" en la que los cuidadores proveen la dirección y estructura, y responden a los señales de hambre y saciedad de los niños, promueve la autorregulación y la responsabilidad de los niños para una alimentación sana. En este artículo, damos recomendaciones para incorporar la alimentación responsiva y modelar conductas alimentarias saludables en las intervenciones nutricionales.
\end{abstract}

Palabras clave: Nutrición Infantil; Conducta alimentaria; Alimentación; Desnutrición; Obesidad (fuente: DeCS BIREME).

\begin{abstract}
Interventions to prevent malnutrition or overweight in children focus on the diet, and give little attention to the behaviors of their caretakers. In their first two years of life, children adopt practices that are embedded in their environment and the behaviors of their caretakers, thus turning into nutrition patterns that will persist during their lifetimes. Therefore, children and caretakers establish a relationship in which they recognize, construe and respond to verbal and non verbal communication signs. Feeding a child by adopting a "responsive" behavior in which caretakers provide guidance and structure, and respond to children's signs of hunger and satiety promotes self-regulation and children's awareness of healthy nutrition. In this article, we give recommendations to include responsive nutrition and model healthy eating behaviors in nutritional interventions.
\end{abstract}

Key words: Infant nutrition; Feeding behavior; Feeding; Malnutrition; Obesity (source: MeSH NLM).

\section{INTRODUCCIÓN}

Las desviaciones en el crecimiento temprano, ya sea por el bajo peso y el retardo del crecimiento o por sobrepeso y obesidad, pueden minar la salud de los niños y, por ende, su bienestar. En muchas familias existen ambas condiciones, por lo que se plantean desafíos a las políticas y directrices nacionales de nutrición. Aunque el papel de la familia es bien conocido (1) muchas de las intervenciones nutricionales se centran casi exclusivamente en la dieta, con escasa atención a los comportamientos de interacción entre los cuidadores y los niños, actividades que caracterizan a las primeras experiencias de alimentación. Los hábitos establecidos tempranamente en la vida suelen persistir con el paso del tiempo ${ }^{(2)}$, esto hace a los primeros años de vida un tiempo ideal para ayudar a las familias a establecer hábitos saludables de alimentación y evitar tanto la desnutrición como el sobrepeso. Este artículo examina el desarrollo de conductas de alimentación temprana y propone estrategias para asegurar un crecimiento y desarrollo óptimos de los niños. 


\section{EPIDEMIOLOGÍA DE LA DESNUTRICIÓN Y LA OBESIDAD: RECOMENDACIONES NUTRICIONALES}

Una inadecuada ganancia de peso puede comenzar antes del nacimiento, como retardo del crecimiento intrauterino, o después del nacimiento, dando lugar a retardo del crecimiento temprano en la vida ${ }^{(3)}$. En algunos países, y en algunos lugares de la sierra rural del Perú, la desnutrición crónica es cercana al $45 \%$ en niños menores de 5 años de edad (4). Muchos niños con desnutrición crónica mantienen su baja estatura durante toda la vida ${ }^{(5)}$ y están en riesgo de un pobre rendimiento escolar durante la niñez, y baja productividad en la edad adulta ${ }^{(6)}$. Las recomendaciones para evitar la desnutrición se centran generalmente en asegurar que los niños reciban una alimentación complementaria rica en nutrientes ${ }^{(7)}$.

La obesidad en niños se ha convertido en un problema global que aumenta el riesgo de los niños de sufrir problemas cardiovasculares, diabetes, el estigma, y problemas de salud mental. Laganancia de peso excesivo antes de los 5 años de edad a menudo se mantiene en la adolescencia ${ }^{(8,9)}$, aumentando el riesgo de obesidad relacionada con las comorbilidades en el futuro. El empeoramiento de los indicadores de salud se atribuye parcialmente a los cambios en la dieta, evidenciada en el consumo de alimentos con alto contenido de azúcar y grasas, y bajo consumo de alimentos ricos en nutrientes, situación producida en los niños durante las últimas décadas ${ }^{(10)}$, lo cual enfatiza la necesidad de intervenir de manera temprana en la vida. Las recomendaciones para la prevención de la obesidad incluyen el aumento del consumo de frutas y verduras; la reducción del consumo de energía (calorías) provenientes de azúcar y grasas saturadas; el aumento de la actividad física moderada y vigorosa, y la reducción del tiempo frente a la pantalla del televisor o de los videojuegos.

\section{LAS NECESIDADESNUTRICIONALES Y LAS HABILIDADES DE DESARROLLO DE LOS BEBÉS}

Las necesidades nutricionales de los infantes son altas para sostener su rápido crecimiento. Se recomienda la lactancia materna exclusiva durante los primeros seis meses de vida ${ }^{(11)}$, la cual no solo satisface las necesidades nutricionales de los bebés, sino que también proporciona inmunidad, permite a los niños a regular su consumo y experimentar la alimentación temprana en el contexto de una relación que nutre su desarrollo; adicionalmente, la lactancia se ha asociado con la reducción de la obesidad y otras enfermedades crónicas ${ }^{(11,12) .}$

El hambre y la saciedad de los niños son guiadas por los procesos internos de regulación e influenciadas por los cuidadores y el contexto de la alimentación (13). ¿Qué?, ¿cuándo? y ¿cómo? comen los niños, en gran medida son establecidos por un amplio rango de determinantes y procesos familiares que se extienden desde las creencias y patrones culturales sobre la comida para los niños; a la disponibilidad y accesibilidad de los alimentos; al tiempo necesario para preparar y servir la comida; a las preferencias del gusto y contexto de los cuidadores $\mathrm{y}$, finalmente, a las percepciones y preocupaciones con respecto al tamaño de los niños, su salud, preferencias, y destrezas de alimentación ${ }^{(14)}$.

Aprender a comer requiere una compleja integración de habilidades en el proceso en lo cual los infantes aprenden a autorregular sus estados de hambre y saciedad en el "dar y recibir " que caracteriza a las interacciones durante los momentos de comer con su cuidador. Aunque las destrezas de alimentarse continúan por un camino de desarrollo claro, hay grandes diferencias individuales en las habilidades y preferencias. El primer año de vida está marcado por un crecimiento rápido y altos requerimientos nutricionales. Como las habilidades orales, motoras y digestivas de los niños maduran, su dieta y comportamiento alimentario atraviesan por grandes transiciones. Ellos pasan de una dieta de leche materna o una dieta basada en leche artificial a los alimentos complementarios y, finalmente, a la dieta familiar. Al mismo tiempo, avanzan desde la dependencia de ser alimentado en una posición supina o semirreclinada a alimentarse en una posición sentada. A la edad de 12 meses, los niños pueden sentarse de forma independiente, masticar y pasar una gama de texturas así como alimentarse a sí mismos con alimentos que pueden recoger con los dedos, y participar en las comidas familiares ${ }^{(14)}$. El impulso hacia la autonomía característica de los niños 1 a 2 años se evidencia en el deseo de los niños pequeños a tocar sus alimentos y comenzar a alimentarse por sí solos ${ }^{(14)}$.

Durante el primer año, los niños y los cuidadores establecen una relación en la que ambos reconocen e interpretan las señales de comunicación tanto verbal como no verbal. Este proceso recíproco constituye una base para la vinculación emocional o de apego entre niños y sus cuidadores que es esencial para el funcionamiento social saludable ${ }^{(15)}$. El rompimiento de su comunicación, caracterizada por interacciones inconsistentes, no-responsivas, pueden minar su relación, lo que lleva a una falta de confianza y seguridad, con el resultado de que 
Tabla 1. Estilos de dar de comer a los niños.

\begin{tabular}{|c|c|c|c|}
\hline \multicolumn{4}{|c|}{ CALIDEZ/APOYO/AFECTO } \\
\hline \multirow{4}{*}{ 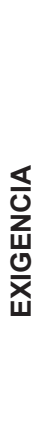 } & & ALTO & BAJO \\
\hline & ALTO & $\begin{array}{l}\text { ESTILO } \\
\text { RESPONSIVO } \\
\text { - } \text { Responde a } \\
\text { señales } \\
\text { - Nutre/Enseña } \\
\text { - Estructura definida }\end{array}$ & $\begin{array}{l}\text { ESTILO } \\
\text { CONTROLADOR } \\
\text { - } \text { Controla/Presiona } \\
\text { - } \text { Rígido } \\
\text { - } \text { Restrictivo }\end{array}$ \\
\hline & & $\begin{array}{l}\text { ESTILO } \\
\text { INDULGENTE }\end{array}$ & $\begin{array}{l}\text { ESTILO NO } \\
\text { INVOLUCRADO }\end{array}$ \\
\hline & BAJO & $\begin{array}{ll}\text { - } & \text { Acepta } \\
\text { - Nutre/Educa } \\
\text { - } & \text { No estructurado }\end{array}$ & $\begin{array}{l}\text { - No involucrado } \\
\text { - Insensible } \\
\text { - No estructurado }\end{array}$ \\
\hline
\end{tabular}

la alimentación puede llegar a ser una ocasión para batallas improductivas por la comida.

Evidencia de la Organización Mundial de la Salud ha demostrado consistencias globales en el crecimiento y desarrollo del niño ${ }^{(16)}$. Expertos en nutrición y en desarrollo infantil han proporcionado directrices universales sobre qué y cómo alimentar a los lactantes y niños pequeños ${ }^{(7)}$. La alimentación receptiva o interactiva se ha convertido en un principio ampliamente recomendado en el que los cuidadores proporcionan la dirección y la estructura (por ejemplo, elección de los alimentos) que los niños requieren para un crecimiento sano, junto con la capacidad de responder a las señales de hambre y saciedad de los niños que promueve la autorregulación y, eventualmente, la responsabilidad de los niños para una alimentación saludable (Tabla 1).

\section{ESTILOS DE DAR DE COMER A LOS NIÑOS}

La ingesta dietética de los niños está influenciada tanto por el clima emocional durante la alimentación (estilos de alimentación) como las prácticas o comportamientos específicos de alimentación. Los estilos de alimentación tienen sus raíces conceptuales en los estilos de crianza de los hijos ${ }^{(17,18)}$, un paradigma basado en el afecto y las exigencias de la interacción cuidador-niño. En los estudios sobre estilos de crianza, la combinación de mucha afecto/alta exigencia, conocido como capacidad de responder, se considera que favorece el desarrollo de la capacidad de regulación de los niños. Estudios observacionales han demostrado que la crianza responsiva (definida como aquella que responde a las señales emitidas por el niño) se relaciona con el desarrollo cognitivo y socioemocional positivo en los niños pequeños (19); por otra parte, ensayos de intervención han demostrado que el mecanismo que vincula la crianza responsiva y el desarrollo de los niños opera a través de las interacciones responsivas entre padres/madres e hijos ${ }^{(20)}$.

La capacidad de responder se caracteriza por un clima emocional en el que las expectativas son claras y los niños experimentan niveles altos de afecto y aceptación por parte de sus cuidadores en respuesta a sus señales. En otras palabras, los cuidadores responden a los señales de los niños de una manera que es, a la vez, rápida y sensible ${ }^{(21)}$, aunque no necesariamente consintiendo a las demandas específicas de los niños. El tono emocional positivo mantiene la interacción y permite a los cuidadores progresar con las orientaciones y lenguaje apropiados para su desarrollo. Los niños contribuyen a la interacción, ganando capacidades de regularse y de interacción que promueven el desarrollo positivo.

Los estilos de crianza han sido evaluados a través de reportes del cuidador y estudios observacionales. Aunque se cree que los estilos de crianza son relativamente constantes en el tiempo, existen pocos estudios longitudinales que abarquen múltiples áreas de desarrollo ${ }^{(22) .}$

El concepto de estilos de crianza se ha aplicado a los estilos de alimentación ${ }^{(23,24)}$. Las relaciones entre los estilos de alimentación y los hábitos alimentarios y el crecimiento han sido discrepantes; las relaciones más consistentes que se han visto son para los estilos de alimentación no responsivos ${ }^{(25,26)}$. El estilo con control/ presión/alimentación forzada se ha relacionado con la baja ingesta ${ }^{27)}$; la alimentación restrictiva relacionada con una ingesta excesiva ${ }^{(28)}$, y la alimentación indulgente relacionada con un aumento en la ingesta y ganancia de peso ${ }^{(29)}$. Pero, por el contrario, una intervención implementada a través de establecimientos de salud de MINSA en una comunidad de bajos ingresos de la zona periurbana en Trujillo, Perú, que por un lado promovía una práctica nutricional específica (por ejemplo, dar purés espesos, incluir un alimento especial: hígado, huevo, pescado) vinculado con un beneficio en los niños (por ejemplo, satisfacerlos y alimentarlos) más una estrategia de alimentación responsiva para una alimentación exitosa (por ejemplo, "Enseña a su hijo a comer con amor, paciencia y buen humor") fue eficaz en la prevención de la desnutrición crónica (30).

\section{LAS PRÁCTICAS DE ALIMENTACIÓN}

Las prácticas de alimentación se refieren a los comportamientos específicos que caracterizan a las interacciones durante la alimentación (por ejemplo, 
número de bocados que se ofrecen, la reacción de los padres frente a la aceptación/rechazo del niño, etc.). Las recomendaciones y evaluaciones de las interacciones durante la alimentación a menudo se centran en las prácticas (por ejemplo: cuándo introducir utensilios; qué hacer cuando un niño se niega a comer, etc.). Ha habido relativamente pocos intentos de integrar las prácticas de alimentación con los estilos de alimentación ${ }^{(23)}$ y una falta de directrices claras para los programas de intervención en la promoción de conductas de alimentación saludable y crecimiento.

Los problemas de alimentación son relativamente comunes en los niños pequeños (25-35\%), alcanzando a menudo un máximo a los 24 meses de edad cuando los niños están luchando por la autonomía y la

Tabla 2. Estrategias para una alimentación saludable: lo que el niño y sus padres pueden hacer y qué debe el niño aprender durante las comidas, según su edad.

\begin{tabular}{|c|c|c|}
\hline $\begin{array}{l}\text { Lo que puede hacer } \\
\text { el niño }\end{array}$ & Lo que pueden hacer los padres & Lo que el niño está aprendiendo \\
\hline \multicolumn{3}{|l|}{$\begin{array}{l}\text { Del nacimiento } \\
\text { hasta los seis meses }\end{array}$} \\
\hline $\begin{array}{l}\text { Señalar hambre/sacie- } \\
\text { dad a través de la voz, } \\
\text { expresión facial y accio- } \\
\text { nes }\end{array}$ & $\begin{array}{l}\text { Responder a las señales: } \\
\text { - Dar de comer al niño cuando está con hambre } \\
\text { - Calmar el niño para que se pueda enfocar en comer } \\
\text { - Agarrar/apoyar al niño y establecer contacto ojo a ojo } \\
\text { - Parar de alimentarlo cuando señale que está lleno } \\
\text { - Evitar dar de comer como respuesta a cada llanto - puede ser } \\
\text { que no llore por hambre }\end{array}$ & $\begin{array}{l}\text { - Confiar que los padres van a satisfacer sus } \\
\text { - } \text { Que puede comunicarse } \\
\text { - Que sus padres escuchan } \\
\text { - Que él/ella es importante para sus padres } \\
\text { - Comer con un horario predecible } \\
\text { - Calmarse solo } \\
\text { - Que la leche es para alimentarse, no para } \\
\text { calmarse }\end{array}$ \\
\hline \multicolumn{3}{|l|}{6 a 12 meses } \\
\hline $\begin{array}{l}\text { - Sentarse } \\
\text { - Comer con sus dedos }\end{array}$ & $\begin{array}{l}\text { - Asegurar que el niño esté apoyado y en posición cómoda para } \\
\text { usar sus manos } \\
\text { - Establecer horarios de comida de la familia y hacerlos una ruti- } \\
\text { na } \\
\text { - Iniciar con alimentos semisólidos y darlos con una cuchara. Lue- } \\
\text { go introducir alimentos más espesos y grumosos, luego peda- } \\
\text { zos suaves. } \\
\text { - Ofrecer alimentos seguros que el niño pueda cogerlos con sus } \\
\text { dedos para promover alimentarse por sí mismo } \\
\text { - Usar dos cucharitas, una para el niño y así aprenda a alimentar- } \\
\text { se por sí mismo } \\
\text { Apagar la TV. Usar el tiempo de las comidas para interrelacio- } \\
\text { narse con el niño solamente. }\end{array}$ & $\begin{array}{l}\text { - Alimentarse por sí mismo } \\
\text { - Decidir cuánto comer } \\
\text { - Experimentar sabores y texturas nuevos y } \\
\text { decidir sus favoritos } \\
\text { - Enfocarse en comer únicamente en los tiem- } \\
\text { pos de la comida }\end{array}$ \\
\hline \multicolumn{3}{|l|}{12 a 24 meses } \\
\hline $\begin{array}{l}\text { - Alimentarse por sí mismo } \\
\text { - Comer diferente tipos de } \\
\text { alimentos } \\
\text { - Empezar a usar tenedor, } \\
\text { cucharas etc. - son segu- } \\
\text { ros para el niño. }\end{array}$ & $\begin{array}{l}\text { - Ofrecer 2-3 comidas saludables al día } \\
\text { - Ofrecer 2-3 entrecomidas (refrigerios) saludables al día } \\
\text { - Ofrecer alimentos que lo puede recoger con sus dedos o cucha- } \\
\text { ra/tenedor, masticarlos y tragarlos fácilmente } \\
\text { - Ofrecer utensilios apropiados para el niño y darle ayuda cuando lo } \\
\text { necesita. }\end{array}$ & $\begin{array}{l}\text { - Probar nuevos alimentos } \\
\text { - Hacer cosas por sí mismo } \\
\text { - Pedir ayuda } \\
\text { confiar que sus padres le van a ayudar } \\
\text { cuando necesita }\end{array}$ \\
\hline
\end{tabular}

\section{4 a 36 meses}

- Seleccionar qué alimentos comer entre los que le ofrecen sus padres cuidadores

- Preparar una comida familiar con 2-3 alternativas sanas, incluyendo algunos alimentos que al niño le gusta. Evitar preparar comidas separadas para el niño

- Ofrecer 2-3 entrecomidas saludables/día

- Dar el ejemplo comiendo entrecomidas saludables delante del niño

- Hablar con el niño

- Usar palabras para ex- - Hacerle preguntas y escuchar las explicaciones del niño. presar sus pensamien- - Usar palabras para ayudar al niño a describir ideas, sentimientos y sentimientos

- Estimular el buen comportamiento

- Seleccionar alimentos saludables

Que conozca su propio cuerpo para comer cuando tiene hambre y parar cuando está lleno.

- Nuevas palabras

- Que el tiempo de la comida es divertido

- Que sus ideas son importantes

- Comportarse bien al momento de comer.
- Ayudar durante las comidas
Ofrecer tareas sencillas como poner las servilletas o cucharas sobre la mesa, poner verduras ya cortadas en la ensalada.
- Que es un miembro importante de la familia

- Que es muy capaz

- Que se siente bien ayudando a otros. 
independencia ${ }^{(31)}$. En su intento de hacer las cosas ellos mismos, muchos niños son descritos como "caprichosos", insistiendo en una variedad limitada de alimentos ${ }^{(13)}$. Aunque la mayoría de los problemas son autolimitados, aquellos que persisten pueden socavar el crecimiento y el desarrollo, y dar lugar a problemas de comportamiento o incluso psicopatologías ${ }^{(32)}$.

Estrategias basadas en la familia pueden influir en las prácticas de alimentación de los niños y viceversa ${ }^{(33)}$ (Tabla 2). El establecimiento de rutinas en torno a la hora de comer (por ejemplo, comer en el mismo lugar y al mismo tiempo); el asegurar que los niños estén sentados en una posición de apoyo y cómodo, sin distracciones (por ejemplo, la televisión), y modelar la conducta apropiada en la hora de comer (por ejemplo, opciones saludables que los niños puedan alimentarse por sí mismos), puede minimizar los problemas de alimentación. Los niños pequeños criados por cuidadores que modelan conductas alimentarias saludables, tales como una dieta rica en frutas y verduras - además de otros alimentos nutritivos como son los productos de origen animal - establecen patrones de conductas y preferencias alimentarias que incluyan frutas y verduras ${ }^{(34)}$. En contraste, los niños de madres que modelan hábitos alimenticios poco saludables (es decir, dietas altas en carbohidratos refinados y grasas saturadas) son propensos a desarrollar, en el futuro, dietas poco saludables por ellos mismos ${ }^{(35)}$. Las intervenciones que promueven las prácticas de alimentación dentro de un contexto de un clima emocional apropiado para el desarrollo del niño donde se responde en forma rápida y con sensibilidad aumentan la probabilidad de una interacción responsiva. Es muy importante que los programas nutricionales incorporen los conceptos relacionados con las prácticas y estilos de alimentación, además de los contenidos nutricionales, donde la promoción de una alimentación responsiva, con las sugerencias incluidas en la Tabla 2, van a permitir una alimentación saludable y evitar la ocurrencia de la desnutrición y del sobrepeso y obesidad.

Fuentes de financiamiento: apoyo parcial de un grant del Eunice Shriver National Institute of Child Health and Human Development, R01HD56099.

Conflictos de interés: ambas autoras declaran no tener ningún conflicto de interés.

\section{REFERENCIAS BIBLIOGRÁFICAS}

1. Kral TV, Rauh EM. Eating behaviors of children in the context of their family environment. Physiol Behav. 2010;100(5):567-73

2. Feinstein L, Sabates R, Sorhaindo A, Rogers I, Herrick D, Northstone K, et al. Dietary patterns related to attainment in school: the importance of early eating patterns. J Epidemiol Community Health. 2008;62(8):734-9.

3. Victora CG, de Onis M, Hallal PC, Blössner M, Shrimpton R. Worldwide timing of growth faltering: revisiting implications for interventions. Pediatr. 2010;125(3):e473-80.

4. UNICEF. The state of the world's children 2009. New York: United Nations Children's fund; 2009.

5. Stein AD, Wang M, Martorell R, Norris SA, Adair LS, Bas I, et al. Growth patterns in early childhood and final attained stature: data from five birth cohorts from low- and middleincome countries. Am J Hum Biol. 2010;22(3):353-9.

6. Victora CG, Adair L, Fall C, Hallal PC, Martorell R, Richter L, et al. Maternal and child undernutrition: consequen- ces for adult health and human capital. Lancet. 2008;371(9609):340-57.

7. World Health Organization. Guiding principles for complementary feeding of the breastfed child. Geneva: WHO; 2003.

8. Gardner DS, Hosking J, Metcalf BS, Jeffery AN, Voss LD, Wilkin TJ. Contribution of early weight gain to childhood overweight and metabolic health: a longitudinal study (EarlyBird 36). Pediatrics. 2009;123(1):e67-73.

9. Nader PR, O’Brien M, Houts R, Bradley R, Belsky J, Crosnoe R, et al. Identifying risk for obesity in early childhood. Pediatrics. 2006;118(3):e594-601.

10. Skinner JD, Ziegler P, Pac S, Devaney B. Meal and snack patterns of infants and toddlers. J Am Diet Assoc. 2004;104(1 Suppl 1):s65-70.

11. American Academy of Pediatrics. Breastfeeding and the use of human milk. Pediatrics. 2005; 115:496-506.

12. Grummer-Strawn LM, Mei Z; Centers for Disease Control and Prevention Pediatric Nutrition Surveillance System. Does breastfeeding protect against pediatric overweight? Analysis of longitu- dinal data from the Center for Disease Control and Prevention Pediatric Nutrition Surveillance System. Pediatrics. 2004;113(2):e81-6.

13. Birch LL. Appetite and eating behavior in children. Pediatr Clin North Am. 1995;42:931-53.

14. Black MM, Hurley K. Infant nutrition. In: Wachs T (ed.). Handbook on Infant Development. Volume 2: Applied and Policy Issues. 2nd ed. NY: Wiley-Blackwell; 2011. p. 33-61.

15. Ainsworth MDS, Blehar MC, Waters E, Wall S. Patterns of attachment: A psychological study of the strange situation. Hillsdale, N.J.: Erlbaum; 1978.

16. de Onis M, Garza C, Onyango AW, Borghi E. Comparison of the WHO child growth standards and the CDC 2000 growth charts. J Nutr. 2007;137(1):144-8.

17. Baumrind D. Current patters of parental autority. Developmental Psychology. 1971;4(1, Pt.2):1-103.

18. Maccoby E, Martin J. Socialization in the context of the family: Parent-child interaction. In: Hetherington EM (ed.). Handbook of child psychology: 
Socialization, personality, and social development. Vol. 4. New York: Wiley; 1983. p. 1-101.

19. Landry SH, Smith KE, Swank PR. Responsive parenting: Establishing early foundations for social, communication, and independent problem-solving skills. Dev Psychol. 2006;42(4):627-42.

20. Landry SH, Smith KE, Swank PR, Zucker T, Crawford AD, Solari EF. The effects of a responsive parenting intervention on parent-child interactions during shared book reading. Dev Psychol. 2012;48(4):969-86.

21. Bornstein MH, Tamis-LeMonda CS. Maternal responsiveness and infant mental abilities: specific predictive relations. Infant Behav Devel. 1997;20:28396.

22. Black MM, Hutcheson JJ, Dubowitz $\mathrm{H}$, Berenson-Howard J. Parenting style and development status among children with non-organic failure to thrive. J Pediatr Psychol. 1994;19(6):689-707.

23. Black MM, Aboud FE. Responsive feeding is embedded in a theoretical framework of responsive parenting. J Nutrition. 2011;141(3):490-4.

24. Hughes SO, Power TG, Orlet Fisher J, Mueller S, Nicklas TA. Revisiting a neglected construct: parenting styles in a child-feeding context. Appetite. 2005;44(1):83-92.

25. Hurley KM, Cross MB, Hughes SO. A systematic review of responsive feeding and child obesity in high-income countries. J Nutr. 2011;141(3):495-501.

26. DiSantis KI, Hodges EA, Johnson SL, Fisher JO. The role of responsive feeding in overweight during infancy and toddlerhood: a systematic review. Int J Obes (Lond). 2011;35(4):480-92.

27. Patrick H, Nicklas TA, Hughes SO, Morales M. The benefits of authoritative feeding style: Caregiver feeding styles and children's food consumption patterns. Appetite. 2005;44(2):243-9.

28. Birch LL, Fisher JO, Davison KK. Learning to overeat: maternal use of restrictive feeding practices promotes girls' eating in the absence of hunger. Am J Clin Nutr. 2003;78(2):215-20.

29. Hughes SO, Shewchuk RM, Baskin $\mathrm{ML}$, Nicklas TA, Qu H. Indulgent feeding style and children's weight status in preschool. J Dev Behav Pediatr. 2008;29(5):403-410.

30. Penny ME, Creed-Kanashiro HM, Robert RC, Narro MR, Caulfield LE, Black RE. Effectiveness of an educational intervention delivered through the health services to improve nutrition in young children: a clusterrandomised controlled trial. Lancet. 2005;365(9474):1863-72.

31. Linscheid TR, Budd KS, Rasnake LK. Pediatric feeding disorders. In: Roberts MC, ed. Handbook of pediatric psychology. 2nd ed.. New York: Guilford Press; 1995.
32. Keren M, Feldman R, Tyano S. Diagnoses and interactive patterns of infants referred to a community-based infant mental health clinic. J Am Acad Child Adolesc Psychiatry. 2001;40(1):27-35.

33. Skinner JD, Carruth BR, Houck KS, Coletta F, Cotter R, Ott D, et al. (1997). Longitudinal study of nutrient and food intakes of infants aged 2 to 24 months. J Am Diet Assoc. 1997;97(5):496-504.

34. Skinner JD, Carruth BR, Bounds W, Ziegler P, Reidy K. Do food-related experiences in the first two years of life predict dietary variety in schoolaged children? J Nutr Educ Behav. 2002;34(6):310-5.

35. Papas M, Hurley K, Quigg A, Oberlander S, Black M. Low-income African American adolescent mothers and their toddlers exhibit similar dietary variety patterns. J Nutr Educ Behav. 2009;41(2):87-94.

Correspondencia: Maureen M. Black Dirección: 727 W. Lombard Street, Room 161, Baltimore, MD 21201 EE.UU.

Teléfono: (01) 410-706-2136 - Fax: 410706-5090

Correoelectrónico:mblack@peds.umaryland. $e d u$

\section{Consulte la versión electrónica de la} Revista Peruana de Medicina Experimental y Salud Pública en

\section{wWW.scopus.com}

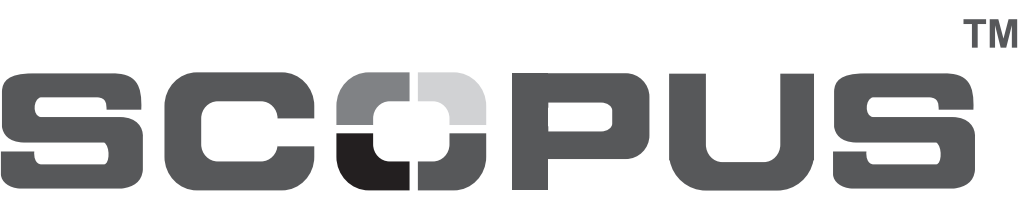

